\title{
VCTA: A Via-Configurable Transistor Array Regular Fabric
}

\author{
Marc Pons*, Francesc Moll* ${ }^{*}$ Antonio Rubio*, Jaume Abella ${ }^{\dagger}$, Xavier Vera ${ }^{\ddagger}$ and Antonio González ${ }^{\ddagger}$ \\ *Universitat Politècnica de Catalunya, Electronic Engineering, \{mpons,moll,rubio\} @ eel.upc.edu \\ †Barcelona Supercomputing Center (BSC-CNS), jaume.abella@bsc.es \\ ${ }_{\ddagger}^{\ddagger}$ Intel Barcelona Research Center, Intel Labs - UPC, \{xavier.vera,antonio.gonzalez\}@intel.com
}

\begin{abstract}
Layout regularity is introduced progressively by integrated circuit manufacturers to reduce the increasing systematic process variations in the deep sub-micron era. In this paper we focus on a scenario where layout regularity must be pushed to the limit to deal with severe systematic process variations in future technology nodes. With this objective, we propose and evaluate a new regular layout style called Via-Configurable Transistor Array (VCTA) that maximizes regularity at device and interconnect levels. In order to assess VCTA maximum layout regularity tradeoffs, we implement 32-bit adders in the $90 \mathrm{~nm}$ technology node for VCTA and compare them with implementations that make use of standard cells. For this purpose we study the impact of photolithography proximity and coma effects on channel length variations, and the impact of shallow trench isolation mechanical stress on threshold voltage variations. We demonstrate that both variations, that are important sources of energy and delay circuit variability, are minimized through VCTA regularity.
\end{abstract}

\section{INTRODUCTION}

As we enter the deep sub-micron era, integrated circuit manufacturers are facing the increasing systematic process variations that arise from the optical lithography manufacturing process. $193 \mathrm{~nm}$ light sources are still used to print critical dimensions of $65 \mathrm{~nm}, 45 \mathrm{~nm}$ and $32 \mathrm{~nm}$, resulting in geometrical layout variability that leads to variations on the electrical characteristics of devices and interconnections.

Important sources of these variations are lithography imperfections such as proximity and coma effect that cause MOS channel length variations. Their impact on delay and leakage current have been demonstrated in [1] where a methodology is described to include layout-dependent variations in static timing analysis and to reduce manufacturing risk.

Resolution Enhancement Techniques (RETs) such as phase shift mask, optical proximity correction and off-axis illumination have been used to greatly improve layout printability and to correct lithography imperfections. However, these techniques are computationally expensive and very timeconsuming for large integrated circuits with arbitrary layout patterns. Thus, layouts with a reduced number of patterns are desirable.

Another source of variation is mechanical stress due to shallow trench isolation. The shape of the oxide diffusion area as well as the location of the MOS device inside this area impact threshold voltage and produce circuit performance variation [2].
New design for manufacturability regularity-based techniques with fewer layout patterns are emerging as a possible solution for manufacturers [3]-[5]. Examples of the use of dummy features to increase layout regularity can be found in [6] showing that regularity is being progressively introduced by Intel or AMD. Regular structuctures for transistors also using dummys reduce the stress-induced performance variations [7]. Other works at Tela Innovations using gridded design rules have been shown to reduce gate critical dimension variability by $4 \mathrm{x}$ to $16 \mathrm{x}$ by improving polysilicon regularity [8]. However, the resulting layouts for these cases are not completely regular. As performance is usually worsen by regularity, layouts are tuned including only the regularity needed to reduce the process variations for the nowadays technology to achieve acceptable yields. In the future, more comprehensive regularity-based techniques will be required to deal with the increasing process variations.

In this paper, we propose and study a new regular design style called VCTA, that stands for Via-Configurable Transistor Array, whose purpose is to push to the limit layout regularity for devices and interconnects to minimize the amount of systematic process variations. Tradeoffs involved in the design of VCTA-based circuits are carefully evaluated.

The structure of the paper is as follows. In section II we briefly describe existent regularity-based techniques and their trade-offs. In section III we detail our VCTA proposal and describe the VCTA basic cell. In section IV we explain VCTA complex circuit layout generation. In section $\mathrm{V}$ we present electric simulations for two 32-bit adders to evaluate the overheads introduced by regularity in area and performance. In section VI we study the benefits of regularity on process variations. Finally, in section VII we provide the conclusions.

\section{REGULARITY-BASED TECHNIQUES OVERVIEW}

Regular designs are based on the repetition of a small set of basic blocks and layout patterns. The main benefit of layout regularity is the reduction of the amount of systematic process variations by allowing RETs to more effectively mitigate lithography printability issues. This translates into a reduction of design cost achieved through (i) a reduction of the yield loss associated to circuit energy and delay unpredictability due to the reduction of process variations, and (ii) a reduction of the time-to-market by accelerating RETs and also due to the lower 


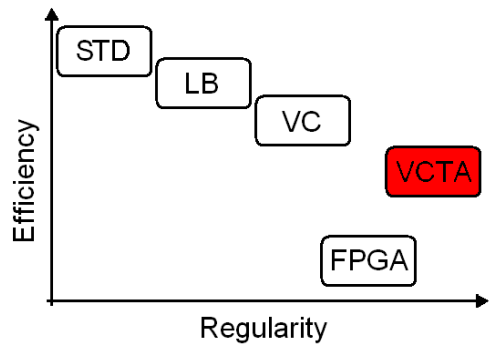

Fig. 1. Regularity vs Efficiency for different layout techniques. STD = Standard Cell, LB = Logic Bricks, VC = Via Configurable Blocks, FPGA = Field-Programmable Gate Array, and our proposal VCTA = Via-Configurable Transistor Array.

number of basic cells or layout patterns and neighborhoods to be optimized.

Among these comprehensive regularity-based techniques there are already some proposals like Logic Bricks (LB), structured ASIC Via-Configurable Logic Blocks (VC) and also Gate Arrays (GA).

The LB design technique is an evolution of the standard cell approach [9]. The basic idea is to find the reduced set of standard cells that are needed for the function to be implemented and optimizing them by reducing the large amount of neighborhood configurations in a standard cell library.

The emerging structured ASICs are constructed using an array of identical basic tiles that contain the logic [10]. The different types of structured ASICs can be classified depending on their regularity granularity defined by the elements that compose the tile. For instance, the tile can be composed by gates, multiplexers, lookup tables, buffers, etc. The condition that has to be ensured is that all functions can be synthesized with the elements included in the tile. VC proposal is to consider a tile composed by via-configurable logic blocks [11]. It consists of two types of blocks: a via-configurable functional cell, containing the combinational logic needed for functions, and two via-configurable inverter arrays, containing inverters to perform the buffer connections with the surrounding tiles.

Among GA, the most evolved design technique is FieldProgrammable Gate Arrays (FPGAs). The basic structure is conformed by logic blocks, including lookup tables and flipflops plus the programming overhead, that are interconnected using routing blocks, consisting of connection boxes and switch boxes [12].

Each of the previous techniques shows different degrees of layout regularity but none of them explores the possibility of maximizing layout regularity both at transistor and interconnect levels.

\section{VCTA PROPOSAL AND BASIC CELL}

The objective of our Via-Configurable Transistor Array proposal (VCTA) is maximizing regularity for devices and interconnects. VCTA uses a single basic cell which is repeated along the circuit. Based on the observation that regular designs such as SRAMs get to the market long before irregular conventional logic designs such as microprocessors [13], [14],
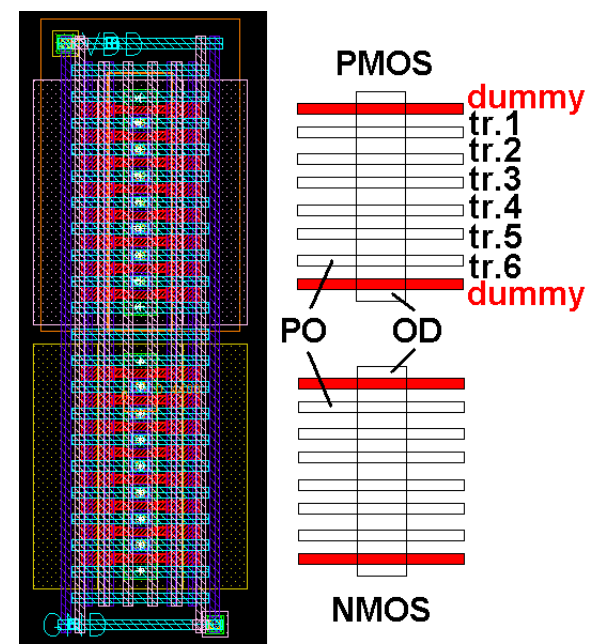

Fig. 2. Transistor Array structure $(\mathrm{PO}=$ polysilicon, $\mathrm{OD}=$ oxide diffusion $)$.

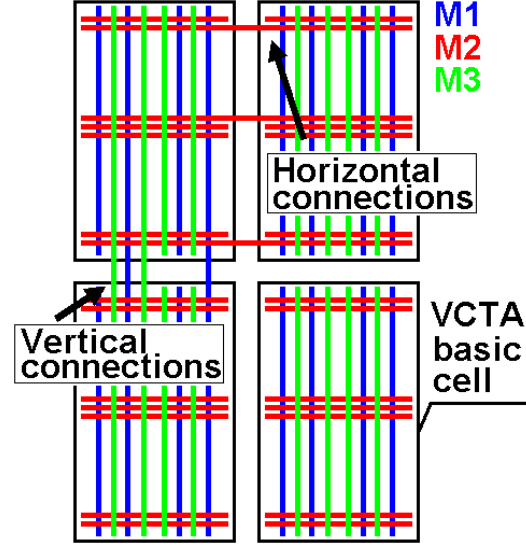

(a)

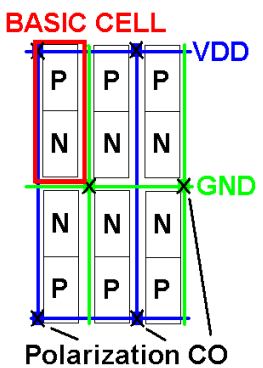

(b)
Fig. 3. (a) Metal grid structure for inter-cell routing (b) Placement and local power supply network of VCTA basic cells ( 6 cells in the picture).

we expect VCTA regularity to reduce the time-to-market, providing a reduction of the amount of systematic process variations with the associated yield loss reduction. However, in general, more regularity implies less efficiency in terms of energy, delay and area.

To summarize the pros and cons of the different existent regular design techniques and to illustrate the expected behavior of our VCTA proposal, we depict in Figure 1 the tradeoff for these designs between design efficiency and regularity.

VCTA is a very fine-grain regular structure, that maximizes layout regularity by setting up regular interconnects and enforces all transistors to have the same dimensions.

\section{A. Maximizing regularity at transistor Level: the Transistor Array}

The Transistor Array is composed of 2 vertically aligned blocks of a number $T$ of PMOS and $T$ NMOS transistors (Figure 2 where $T=6$ ). Note that the $T$ transistors in each case share the same oxide diffusion in order to increase transistor density and thus reduce the area of the basic cell. With this 


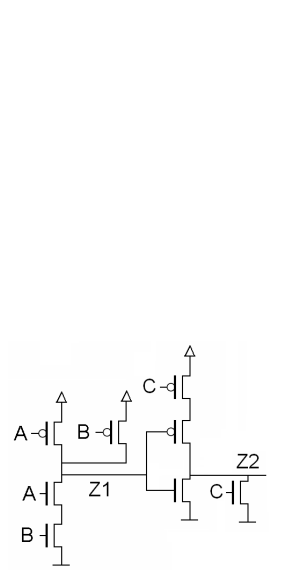

(a)

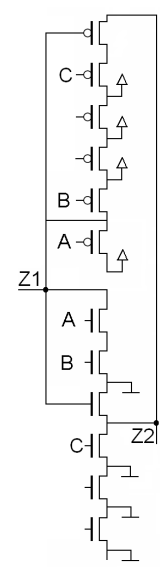

(b)

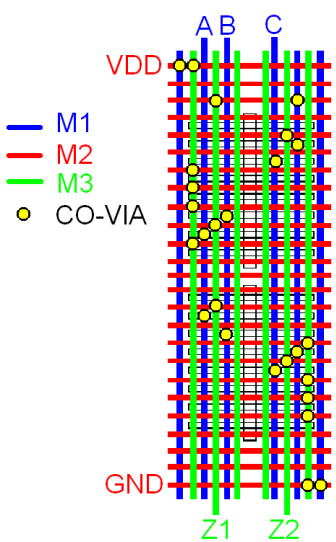

(c)
Fig. 4. NAND-NOR: (a) Schematic (b) VCTA schematic (c) VCTA layout.

constraint VCTA transistors are connected in series by default. However, we can implement parallel connections by properly setting up vias as it will be explained in the next subsections. In order to force maximum transistor layout regularity, all transistors have the same width and the minimum channel length. To further reduce process variations, we add 2 dummy transistors (the ones on the upper and lower extremes). In this way we avoid possible variations in drains/sources between two polysilicon gates and drains/sources at the edges with only one gate on one side.

\section{B. Maximizing regularity at interconnect level: the Via- Configurable choice}

In order to ensure interconnect regularity VCTA uses a regular interconnect grid of parallel metal lines. The lines alternate from horizontal to vertical direction from one layer to the next. Intra-cell routing is performed by means of a viaconfigurable structure where all contacts and vias are placed depending on the function to be synthesized. Inter-cell routing between VCTA basic cells is achieved by the extension of the metal lines across the borders of the VCTA cells. Contacts, vias and inter-cell metal interconnections are the only source of layout irregularity of our VCTA design.

\section{Implementation of VCTA}

In order to study VCTA designs we will use a particular implementation with $T=6$ in the rest of the paper. The choice of having 6 PMOS and 6 NMOS transistors in the basic cell is related to the possibility of implementing 2 logic branches of transistors with a maximum length of 3 serial transistors to avoid body effect and excessive serial resistance issues. All transistors have minimum channel length and $440 \mathrm{~nm}$ width ensuring enough transistor strength to drive the associated parasitics of the dense interconnect metal grid structure.

The via-configurable interconnection scheme uses three metal levels, M1 to M3, forming a regular routing grid where M1 and M3 wires are vertical and M2 wires are horizontal. The choice is based on using the lowest metal levels.

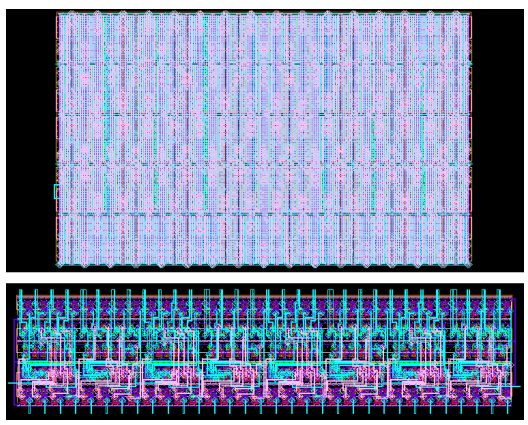

Fig. 5. Layouts of CLA32 VCTA $(65.5 \mu \mathrm{m} \times 40 \mu \mathrm{m}$, top) and STD $(80 \mu \mathrm{m}$ x $17.4 \mu \mathrm{m}$, bottom)

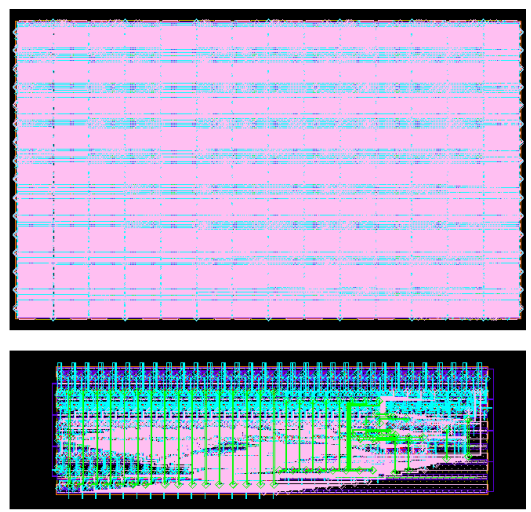

Fig. 6. Layouts of KS32 VCTA $(110 \mu \mathrm{m} \times 64 \mu \mathrm{m}$, top) and STD $(91 \mu \mathrm{m} \times$ $29.5 \mu \mathrm{m}$, bottom)

Regarding the intra-cell connections, we use PO-M1 contacts to configure the transistor gate inputs, M1-M2 vias to configure the basic cell inputs and outputs and finally M2-M3 vias to configure the parallel transistor connections. Figure 4 shows an example of intra-cell connections using contacts and vias (CO-VIA) to implement a simple NAND-NOR gate inside of a basic cell. Note that we still have spare transistors (whose drain and sources are connected to power supply in order to avoid undesirable energy consumption) that we can use to implement other functions if necessary.

We use M1 and M3 layers for vertical inter-cell connections and M2 for horizontal inter-cell connections (Figure 3a).

Note that we can consider many other VCTA implementations with different number of transistors, metal layers, etc. However, such a study is out of the scope of this paper.

\section{COMPleX CiRCUits With VCTA}

In order to illustrate that our VCTA regular design technique allows the implementation of complex circuits we have implemented binary adders, a common block in integrated circuit designs. In particular, we have developed complete layouts in the $90 \mathrm{~nm}$ technology node for a 32-bit Carry-Lookahead adder (CLA32) and for a 32-bit Kogge-Stone adder (KS32) using the VCTA structure and also the Standard Cell approach (STD) to evaluate the area, energy and delay overheads in those commonly used circuits. 


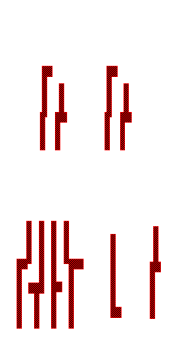

(a)

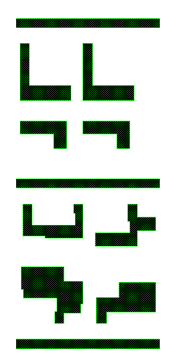

(b)

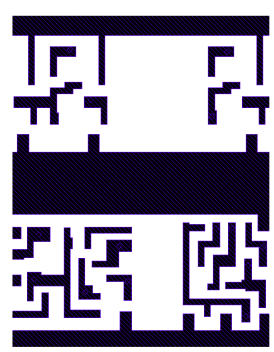

(c)
Fig. 7. STD CLA32 Layout Layer Masks (a) PO (b) OD (c) M1.

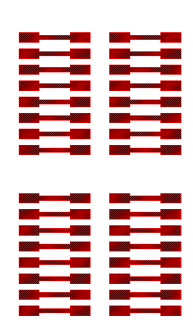

(a)

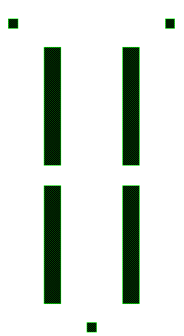

(b)

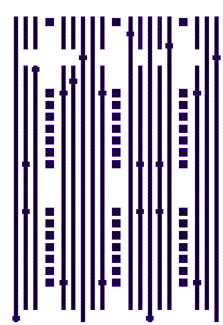

(c)
Fig. 8. VCTA CLA32 Layout Layer Masks (a) PO (b) OD (c) M1.

The resulting complete layouts captures are presented for the CLA32 in Figure 5 and for the KS32 in Figure 6.

The steps that we have followed for VCTA layout generation are: (1) find out the logic functions needed to implement the structure of the circuit, (2) map the transistors of these functions into the VCTA basic cell as we have shown in the previous section for the NAND-NOR gate, (3) manually place and route them to obtain the complete layout. The automation of the whole VCTA design flow is part of our future work. For the STD layout generation, we have used the public standard cell layouts provided in [15] that offers a complete set of portable CMOS libraries.

The binary adder circuits studied require 6 different types of logic functions: an inverter, an XOR, a 2-input NAND, a 4-input NAND, an AND-OR and an OR-AND [16]. We have mapped these functions into the VCTA basic cells. In some cases we were able to implement 2 functions into a single VCTA basic cell. This can be done when the functions are next to each other in the circuit (e.g., the output of one of the functions is the input of the other one, or they share the same inputs).

As a consequence, the VCTA layouts can be composed by fewer cells than the STD layouts. For instance, the complete CLA32 finally required 228 standard cells and only 160 VCTA basic cells. We have manually placed and routed those VCTA cells trying to minimize the interconnect distances as well as for STD cells.

For the placement of our basic cells we have also considered the local power supply network symmetries. We depict the general placement of our basic cells in Figure $3 b$ for 6 basic cells. In the VCTA basic cell we reserve M1 and M3 wires in
TABLE I

RESULTS WITHOUT PROCESS VARIATIONS $($ WCD $=$ WORST-CASE DELAY, AVGE $=$ AVERAGE ENERGY $)$

\begin{tabular}{|c|c|c|c|}
\hline & WCD(ns) & AVGE(pJ) & Area $\left(\mu \mathrm{m}^{2}\right)$ \\
\hline \hline CLA32 STD & 1.11 & 0.21 & 1394 \\
\hline CLA32 VCTA & 2.15 & 0.47 & 2620 \\
\hline Ratio & $1.94 \mathrm{x}$ & $2.24 \mathrm{x}$ & $1.88 \mathrm{x}$ \\
\hline \hline KS32 STD & 0.84 & 0.33 & 2684 \\
\hline KS32 VCTA & 1.69 & 0.79 & 7046 \\
\hline Ratio & $2.00 \mathrm{x}$ & $2.39 \mathrm{x}$ & $2.63 \mathrm{x}$ \\
\hline
\end{tabular}

each metal layer for VDD and GND. These wires are shared across neighbor cells. In this way, we can reduce the area when implementing a full circuit with multiple basic cells. We use the same design criterion sharing polarization contacts.

To illustrate the layouts that we have generated, Figures 7 and 8 present CLA32 layout masks for STD and VCTA designs for polysilicon (PO), oxide diffusion (OD) and metal 1 (M1) layers. By visual inspection we can see how our VCTA design is much more regular than the STD approach.

\section{IMPACT ON AREA AND PERFORMANCE}

We have performed complete electrical simulations of the extracted layouts of CLA32 and KS32 in the $90 \mathrm{~nm}$ technology node using the HSPICE simulator. We have evaluated both the adders designed with our VCTA regular design as well as those based on standard cells in terms of delay and energy for 10400 inputs that we have sampled from all 26 programs in the SPEC2000 benchmark suite [17]. We have measured the delay from input variation to the associated output transition considering the cross at $90 \%$ of the voltage rise or fall swings. We have also measured energy for each input combination integrating the current demand at the power supply source during the addition. Finally, we have measured the area directly from the layout. We show measurement results for worst-case delay (WCD) and average energy dissipation (AVGE) for all the inputs in Table I.

First, this particular choice for VCTA regular design implies an increase around $2 \mathrm{x}$ in area (1.88x for CLA32 and 2.63x for KS32) when compared to the STD approach. The area increase is basically due to the regularity requirements and redundancy, because all possible configurations of devices and interconnects are in place in the VCTA basic cell. The basic cell includes dummy transistors, spare transistors and also spare interconnects which increase the total area.

In terms of WCD and AVGE, both CLA32 and KS32 present more than a $2 \mathrm{x}$ energy ratio but around a $2 \mathrm{x}$ delay ratio. In fact overheads introduced by VCTA when compared to STD are very much dependent on the function to implement. STD uses different standard cells depending on the circuit optimization but VCTA always uses the same basic cell. Energy and delay overheads are due to the parasitics introduced by our VCTA metal grid.

Another VCTA parameter to optimize is transistor sizing (all the transistors have the same dimensions). With our present choice of $440 \mathrm{~nm}$ for width, by connecting in parallel 


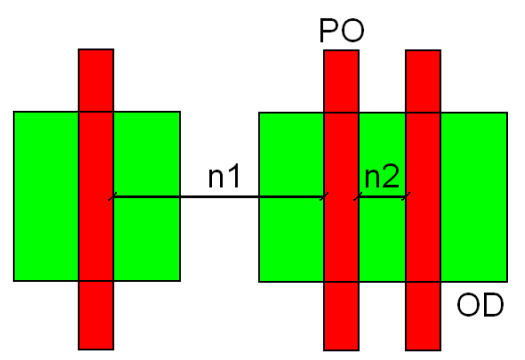

Fig. 9. Proximity and coma effect model measurements

TABLE II

CHANNEL LENGTH VARIATIONS

\begin{tabular}{|c|c|c|}
\hline & Proximity Effect L $3 \sigma / \mu$ & Coma Effect L $3 \sigma / \mu$ \\
\hline \hline CLA32 STD & $5.31 \%$ & $6.19 \%$ \\
\hline KS32 STD & $5.16 \%$ & $6.48 \%$ \\
\hline
\end{tabular}

transistors, we can only emulate wider transistors of $880 \mathrm{~nm}$, $1320 \mathrm{~nm}$, etc., with a width multiple of the basic transistor, and this is not always optimal.

Note also that logic functions implemented such as NAND, XOR, etc. are particularly suitable for STD, but may be suboptimal for VCTA.

\section{IMPACT ON PROCESS VARIATIONS}

Evaluating the impact of layout regularity on systematic process variations is key to demonstrate the usefulness of maximizing layout regularity using VCTA. As variability in printed features depends on their neighborhood, the link between the different shapes in the layout and the amount of process variations has to be studied.

Channel length variations and the impact of mechanical stress on threshold voltage have been evaluated as they are major sources of circuit performance variations.

\section{A. Channel length variations: proximity and coma effect}

Models for systematic variations of channel length (L) variability can be found in [18] taking into account proximity and coma Effects. Basically, proximity and coma effects models associate to each channel a percentage of $\mathrm{L}$ variation depending on the layout neighborhood on both sides of the feature to be printed. The models are based on the inspection of the layout to the left and to the right of the feature in order to define the kind of neighborhood that the channel has. They measure the distances to the first polysilicon line in each direction. Figure 9 depicts an example of distances $\mathrm{n} 1$ to the left and $\mathrm{n} 2$ to the right. The difference between both is that for proximity effect left side and rigth side distances are equivalent in their impact on variations but for coma effect they are not. The models include tables with the nominal amount of process variations for each case and the final percentage variation for $\mathrm{L}$ can be obtained by setting the maximum percentage range of variations. The final result is the expected $\mathrm{L}$ for each of the transistors on the layout. The entire circuit $\mathrm{L}$ distribution can then be characterized by its mean $\mu$ and its standard deviation $\sigma$.

Using those proximity and coma effect models we have measured the L systematic process variations of the adder layouts for VCTA and STD for the different sources of systematic variability considering $10 \%$ maximum $L$ variations.

As all the transistors in the VCTA basic cell have the same layout neighborhood, with two polysilicon lines at the same distance, they are all affected by the same systematic $\mathrm{L}$ variations, thus showing no $\sigma$ in the $\mathrm{L}$ distribution. This is achieved by the use of the dummy polysilicon lines at the edges of the PMOS and NMOS transistor arrays.

On the other hand, STD adders that use different cells with different placements present higher number of layout neighborhoods. The L statistics in terms of $3 \sigma / \mu$ are presented in Table II. For proximity effect, CLA32 and KS32 transistors see 7 and 8 neighborhoods respectively. For Coma Effect, which differentiates the sides for the distances measured, there are 9 and 10. That is why coma effect variability is higher than proximity effect variability.

The final result is that all VCTA transistors are affected by the same $\mathrm{L}$ systematic variation and therefore have all the same $\mathrm{L}$ whereas the $\mathrm{L}$ variability between transistors is around 5-6\% for STD. Therefore, we can conclude that L variations for proximity effect and coma effect are minimized through VCTA regular layout designs.

Note that these results show the regularity of VCTA at two levels. First, the L variations are the same for both CLA32 and KS32 for the VCTA design whereas they depend on the particular circuit for the STD design. This is because VCTA uses the same basic cell for both adders and STD uses different cells. This is VCTA regularity at cell level. Second, VCTA maximizes regularity inside the basic cell and shows only one neighborhood for all transistors whereas STD shows different neighborhoods inside each of the cells. This is VCTA regularity at transistor level.

\section{B. Threshold voltage variations: mechanical stress}

Models for silicon mechanical stress due to Shallow Trench Isolation (STI) are included in the BSIM4 transistor models [19]. Transistor performance is affected depending on the shape of the oxide diffusion area and on the position of the device inside this area. In particular, threshold voltage (Vth) varies depending on the distances from the channel to the edge of the difusion (where the STI begins). Figure 10 shows an example for the measurement of these $\mathrm{d} 1$ and $\mathrm{d} 2$ distances. The relative impact also depends on the dimensions of the transistor. Transistors with wider channel will be less affected.

By extracting these data from the layout and using the models supplied for the $90 \mathrm{~nm}$ technology node, we have calculated the Vth variations for PMOS and NMOS transistors in the CLA32 and the KS32 adders. The results for the VCTA and STD designs are shown in Table III.

For VCTA transistors, there are only three different cases. From Figure 2 it can be seen that transistors 1 and 6 will have the same STI stress because the VCTA basic cell is 


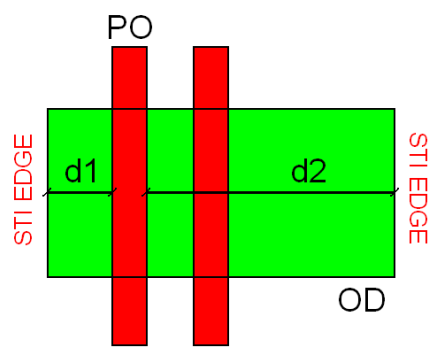

Fig. 10. STI Stress model measurements

TABLE III

THRESHOLD VOLTAGE VARIATIONS

\begin{tabular}{|c|c|c|}
\hline & PMOS Vth $3 \sigma / \mu$ & NMOS Vth $3 \sigma / \mu$ \\
\hline \hline CLA32 STD & $4.85 \%$ & $6.25 \%$ \\
\hline CLA32 VCTA & $0.82 \%$ & $1.24 \%$ \\
\hline Ratio & $0.17 \mathrm{x}$ & $0.20 \mathrm{x}$ \\
\hline \hline KS32 STD & $4.07 \%$ & $5.83 \%$ \\
\hline KS32 VCTA & $0.82 \%$ & $1.24 \%$ \\
\hline Ratio & $0.20 \mathrm{x}$ & $0.21 \mathrm{x}$ \\
\hline
\end{tabular}

symmetric. The same occurs for transistors 2 and 5 and finally for transistors 3 and 4. Furthermore, the VCTA transistors have all the same channel width and therefore will be affected similarly.

On the other hand, for STD, there is a higher number of cases related to the different transistor neighborhoods and to the different transistor sizings. That is why for VCTA the Vth variability is around $1 \%$ and for STD it reaches $4 \%$ for PMOS and 6\% for NMOS. The ratios for the reduction of Vth variability due to VCTA regularity are close to $0.20 \mathrm{x}$.

Again, the results show VCTA regularity at two different levels. First, at cell level we can see how VCTA shows the same Vth variations independently of the circuit considered. Second, at transistor level, the number of cases for STI stress is also reduced because of transistor array regularity.

\section{CONCLUSION}

This paper proposes and evaluates the VCTA design technique to explore the impact of maximizing layout regularity in future technologies that will have to deal with increasing systematic process variations.

CLA32 and KS32 adder layouts using a particular implementation of the VCTA basic cell have been developed to illustrate the VCTA methodology.

VCTA proposal maximizes regularity at cell level using a single basic cell, at transistor level with the Transistor Array structure, and finally at interconnect level with the ViaConfigurable choice.

Lithography models for proximity and coma effects and STI mechanical stress show how VCTA regularity minimizes the channel length and threshold voltage MOS systematic process variations that are affecting STD designs.

\section{ACKNOWLEDGMENT}

This research work has been supported by Intel Corporation, Feder Funds, the Spanish Ministry of Education and Science under grant TIN2007-61763, TEC2008-01856 and FPU AP2007-04125 and the Generalitat de Catalunya under grant 2009SGR1250.

\section{REFERENCES}

[1] M. Choi and L. Milor, "Impact on circuit performance of deterministic within-die variation in nanoscale semiconductor manufacturing," IEEE $T C A D$, vol. 25 , no. 7 , pp. $1350-1367,2006$.

[2] V. Moroz et al., "Stress-aware design methodology," in ISQED, 2006 , pp. 807-812.

[3] B. Wong et al, Nano-CMOS Design for Manufacturability: Robust Circuit and Physical Design for Sub-65 nm Technology Nodes. John Wiley \& Sons, 2009

[4] M. Orshansky et al, Design for Manufacturability and Statistical Design: A Constructive Approach. Springer, 2008.

[5] C. Chiang and J. Kawa, Design for Manufacturability and Yield for Nano-Scale CMOS. Springer, 2007.

[6] J. Dick, "Design-for-manufacturing features in nanometer processes - a reverse engineering perspective," in $A S M C, 2009$, pp. 56-61.

[7] P. G. Drennan et al., "Implications of proximity effects for analog design," CICC, pp. 169-176, 2006.

[8] M. Smayling et al, "Low k1 logic design using gridded design rules," vol. 6925, no. 1. SPIE, 2008.

[9] V. Kheterpal et al, "Design methodology for IC manufacturability based on regular logic-bricks," in DAC, 2005, pp. 353-358.

[10] B. Zahiri, "Structured ASICs: opportunities and challenges," in ICCD, 2003, pp. 404-409.

[11] Y. Ran and M. Marek-Sadowska, "Designing via-configurable logic blocks for regular fabric," IEEE Transactions on VLSI Systems, vol. 14, no. 1, pp. 1-14, 2006.

[12] M. Lin and A. El Gamal, "A routing fabric for monolithically stacked 3d-fpga," in FPGA, 2007, pp. 3-12.

[13] http://www.intel.com/pressroom/.

[14] http://www.tcmagazine.com/comments.php? shownews $=24545$.

[15] G. Petley, VLSI and ASIC Technology Standard Cell Library Design, http://www.vlsitechnology.org.

[16] H. Neil et al, CMOS VLSI Design, A Circuits and Systems Perspective. Pearson, 2005.

[17] http://www.spec.org/cpu2000.

[18] M. Choi and L. Milor, "Diagnosis of optical lithography faults with product test sets," IEEE TCAD, vol. 27, no. 9, pp. 1657-1669, 2008.

[19] http://www-device.eecs.berkeley.edu/ bsim3/bsim4.html. 